\title{
INFLUENCE DE L'INGESTION D'URÉE SUR LA \\ PHYSIOLOGIE DE LA DIGESTION GHEZ LE MOUTON
}

PAR

\section{H. LE BARS et H. SIMONNET}

Laboratoire de recherches de la Chaire d'Anatomie et Physiologie comparées des animaux domestiques.

Institut national Agronomique, Paris.

Du fait de l'existence de la micropopulation des réservoirs gastriques, les Ruminants sont capables, dans certaines conditions, d'utiliser l'urée ou les sels ammoniacaux introduits dans leur alimentation. De nombreuses recherches ont été consacrées aux modalités de cette utilisation et à la valeur de l'urée en tant que source d'azote.

Une des premières questions qui se posent est celle de la toxicité de l'urée chez les Ruminants. L'urée n'est pas toxique par elle-même, mais l'uréase des microorganismes la transforme en ammoniaque qui passe dans le sang et qui peut être à l'origine d'accidents mortels. Les recherches effectuées à ce sujet $(I, 2,3,4)$ ne permettent pas de tirer une conclusion précise sur la toxicité de l'urée administrée par voie buccale chez les Ruminants.

Une série d'essais sur un lot de I $_{5}$ brebis nous a permis d'apporter quelques renseignements complémentaires concernant la toxicité aiguë et la toxicité chronique de cette substance.

Pour étudier la toxicité aiguë, nous avons administré en une seule fois des doses d'urée comprises entre o, I et $4 \mathrm{~g}$ par kilo de poids vif.

Dans le cas de la toxicité chronique, 2 brebis ont reçu chaque jour pendant 2 mois, des quantités d'urée correspondant à I g/kg.

\section{I. - TOXICITÉ AIGÜ̈}

Les animaux recevaient une ration composée de foin de luzerne et de pulpes de betteraves (pulpes desséchées préparées suivant les besoins).

Nous avons utilisé 2 formes d'urée : urée pure et urée technique contenant $97, \mathrm{I}$ p. Ioo d'urée et 2 p. Ioo de biuret.

Annales de Zootechnie. - I959. 
L'administration a été faite en solution aqueuse soit à la sonde œsophagienne, soit par 1'orifice d'une canule de fistule permanente du rumen, soit dans un mélange de pulpe et de farine d'orge consommé spontanément par l'animal.

Certains animaux ont été utilisés plusieurs fois, mais un intervalle d'une huitaine de jours séparait toujours 2 essais consécutifs.

Les résultats obtenus peuvent se classer en 2 groupes suivant que les animaux sont morts ou ont survécu.

\section{A. - Essais mortels.}

Les essais sont résumés dans le tableau suivant:

\begin{tabular}{|c|c|c|}
\hline $\begin{array}{c}\text { Dose administrée } \\
\mathrm{g} / \mathrm{kg}\end{array}$ & Mode d'administration & Durée de survie \\
\hline $\begin{array}{l}\text { Urée pure : } \\
\begin{array}{l}4 \\
3 \\
2,5 \\
2 \\
\mathrm{I}, 5 \\
\mathrm{I} \\
\mathrm{0}, 5\end{array}\end{array}$ & 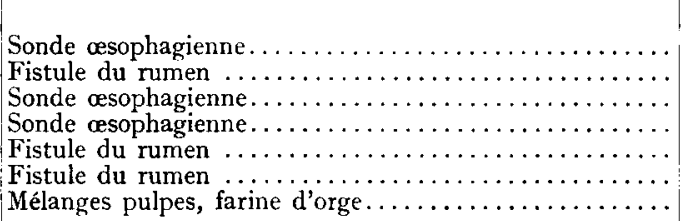 & $\begin{array}{l}40 \text { minutes } \\
\text { Ih, I } 5 \text { minutes } \\
3 \text { heures } \\
2 \text { heures } \\
45 \text { minutes } \\
\text { Ih. } 20 \text { minutes } \\
\text { I heure }\end{array}$ \\
\hline
\end{tabular}

Dans tous les cas, les manifestations cliniques de l'intoxication sont comparables.

Les premiers symptômes se manifestent après un temps de latence de ro à 30 minutes. On note de l'inquiétude, des tremblements, localisés principalement aux muscles des membres ; puis l'animal se couche et on observe à intervalles plus ou moins espacés (5 à 30 minutes) des périodes d'agitation de durée variable. L'agitation peut se traduire soit par des alternatives d'extension et de relâchement des membres (mouvement de pédalage), soit par des contractures toniques ou cloniques. Ces contractions peuvent être généralisées, mais le plus souvent elles sont localisées aux muscles abdominaux.

Les éructations sont fréquentes, la salivation est abondante et des matières alimentaires régurgitées s'écoulent de la cavité buccale. $\mathrm{L}_{\text {a }}$ respiration est dyspnéique, le cœur accéléré, les yeux sont révulsés et la brebis pousse des bêlements plaintifs. La motricité du rumen est inhibée, ce qui entraîne la météorisation.

La mort survient en opistothonos au cours d'une de ces crises, le plus souvent dans l'heure qui suit le début des manifestations cliniques.

L'autopsie ne révèle que des lésions d'asphyxie et en particulier la cyanose des muqueuses. 


\section{B. - Essais non mortels.}

Les résultats précédents montrent que l'on peut tuer un mouton en lui administrant en une seule fois des doses d'urée correspondant à 0,5 et I $\mathrm{g} / \mathrm{kg}$. Ces quantités ne sont cependant pas toujours toxiques ainsi que des quantités supérieures.

C'est ce que montrent les observations suivantes.

\begin{tabular}{|c|c|c|}
\hline \multirow{2}{*}{$\begin{array}{l}\text { Dose administrée ( } 1 \text { ) } \\
\mathrm{g} / \mathrm{kg}\end{array}$} & \multicolumn{2}{|c|}{ Nombre d'observations } \\
\hline & Urée pure & Urée technique \\
\hline 2 & & I \\
\hline $\mathrm{I}, 75$ & I & \\
\hline $\mathrm{T}, 50$ & 2 & $\mathrm{r}$ \\
\hline$T, 25$ & 3 & 3 \\
\hline 1 & 3 & 6 \\
\hline 0,75 & I & \\
\hline 0,50 & 5 & 5 \\
\hline 0,40 & 2 & 2 \\
\hline 0,30 & I & 2 \\
\hline 0,10 & 2 & I \\
\hline
\end{tabular}

(I) Directement dans le rumen par l'orifice de la canule

I1 est intéressant de décrire les essais réalisés avec les doses de $2 \mathrm{~g}$ et $\mathrm{I}, 75 \mathrm{~g} / \mathrm{kg}$ qui ne sont pas nécessairement mortelles mais qui causent des accidents.

a) Une brebis de $34 \mathrm{~kg}$ reçoit $2 \mathrm{~g} / \mathrm{kg}$ d'urée technique administrée en solution aqueuse par l'orifice de la canule du rumen. 37 minutes plus tard, débute l'inhibition de la motricité du rumen qui devient rapidement totale. Une heure après l'administration l'animal présente une phase d'excitation avec respiration dyspnéique, suivie d'un état comateux. Une deuxième phase d'agitation avec chutes répétées, jetage sanguinolent, est observée 2 heures plus tard. I,e retour au calme est progressif et la motricité du rumen ne redevient normale que 9 heures après l'administration.

b) Une brebis de $34 \mathrm{~kg}$ reçoit $\mathrm{I}, 75 \mathrm{~g} / \mathrm{kg}$ d'urée pure dans les mêmes conditions que dans l'essai précédent. L'évolution de l'intoxication est comparable, on note une première phase d'excitation 16 minutes après l'administration, avec chutes répétées, tremblements musculaires et inhibition de la motricité gastrique, une deuxième période d'excitation, une heure après l'administration. Le retour à la normale se fait en $2 \mathrm{~h}$. 15 .

Ces faits mettent donc en évidence des variations individuelles considérables de la sensibilité du mouton à l'urée, même chez des animaux soumis à des régimes alimentaires identiques. Il est donc difficile de déterminer la dose toxique aiguë. Sur 42 essais réalisés avec des doses comprises entre $0, I$ et $I, 5 \mathrm{~g} / \mathrm{kg}$, 3 seulement ont éṫ mortels. I a dose toxique se situe donc approximativement autour de $I, 5 \mathrm{~g} / \mathrm{kg}$, mais la mort peut survenir à $0,5 \mathrm{~g}$ (I cas mortel sur II essais). 


\section{TOXICITÉ CHRONIQUE}

2 brebis, pesant respectivement 43 et $46 \mathrm{~kg}$, consommant à volonté du foin de luzerne, ont reçu chaque jour pendant un mois un mélange ainsi constitué :

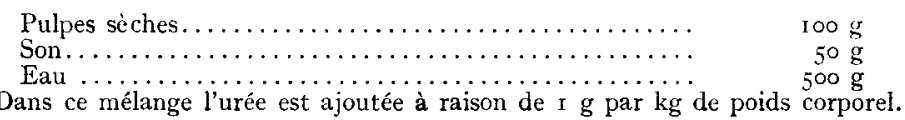

3 semaines après le début de l'expérimentation, le mélange est de plus en plus mal accepté. La ration de fourrage est alors réduite à environ I,5 kg par animal et par jour et ils reçoivent de la paille mélassée à volonté.

A la fin de cet essai, les sujets sont en bon état, ils pèsent respectivement 45 et $46 \mathrm{~kg}$ et n'ont manifesté aucun signe clinique d'intoxication.

Ces 2 brebis sont ensuite soumises à un régime pauvre en protéines (ne contenant que 25 à $30 \mathrm{p}$. Ioo de protéines du régime initial) complété par de l'urée. Chaque animal reçoit par jour :

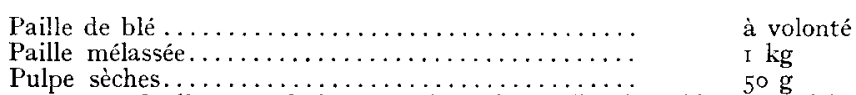

Ils reçoivent en outre de l'urée technique à raison de $\mathrm{i} \mathrm{g} / \mathrm{kg}$ de poids corporel incorporée dans roo $\mathrm{g}$ de paille mélassée.

Ce dernier mélange est donné à part afin de contrôler son ingestion par les brebis.

Après quelques jours d'adaptation à ce nouveau régime, la paille mélassée et le mélange à base d'urée sont bien acceptés. 30 jours plus tard, les 2 brebis ne présentent aucun signe d'intolérance, sont en bon état et pèsent 46 et $47 \mathrm{~kg}$.

Ces résultats confirment la variabilité des effets constatés suivant les animaux. Alors qu'une prise unique d'urée de $0,5 \mathrm{~g}$ à I $\mathrm{g} / \mathrm{kg}$ peut être mortelle chez certains sujets, ces 2 brebis ont bien supporté une dose quotidienne de $\mathrm{I} \mathrm{g} / \mathrm{kg}$ pendant 2 mois. Il faut toutefois remarquer que dans ce dernier cas l'ingestion de l'urée est progressive, l'animal mettant parfois plusieures heures pour absorber la préparation uréique. Dans les expériences de toxicité aiguë au contraire, l'administration est faite rapidement, en quelques minutes. Mais ce facteur n'est pas le setul en cause. En effet, dans un cas (essai mortel à la dose de $0,5 \mathrm{~g} / \mathrm{kg}$ ), un mélange : pulpes et farine d'orge additionné d'urée à la dose de $\mathrm{I} \mathrm{g} / \mathrm{kg}$ avait été préparé ; l'animal a consommé spontanément la moitié de la préparation et est mort une heure plus tard.

Ces constatations montrent donc que l'urée (urée pure ou urée technique) peut être toxique chez les ruminants. La dose administrée doit être 
inférieure à $0,5 \mathrm{~g} / \mathrm{kg}$ et l'ingestion progressive afin d'éviter l'apport massif d'urée dans les réservoirs gastriques. Quelle que soit la quantité ajoutée aux aliments, il est nécessaire de surveiller les animaux car les variations de la sensibilité d'un sujet à l'autre sont considérables.

\section{III. - INFLUENCE DE L'URÉE SUR LA MOTRICITÉ GASTRIQUE}

Au cours des essais d'intoxication aiguë du mouton par l'urée, on observe cliniquement un arrêt de la motricité du rumen. Il était donc intéressant de rechercher l'influence de cette substance, administrée à différentes doses, sur la motricité gastrique.

Cette étude a été réalisée sur 8 sujets $(7$ brebis de réforme pesant de 35 à $60 \mathrm{~kg}$, un agneau de $20 \mathrm{~kg}$ ) portant une fistule permanente du rumen. L'enregistrement des contractions du rumen a été fait sur l'animal non anesthésié selon la technique déjà décrite (5).

Comme dans le cas de l'étude de la toxité aiguë, nous avons utilisé 2 formes d'urée : urée pure et urée technique contenant 97, I p. roo d'urée.

Les animaux recevaient par jour la ration suivante :

fourrage de luzerne à volonté ;

$200 \mathrm{~g}$ de pulpes de betteraves desséchées (préparées suivant les besoins);

$50 \mathrm{~g}$ de farine d'orge.

Dans tous les cas, l'urée a été introduite en solution aqueuse par l'orifice de la canule gastrique.

Les résultats obtenus sont résumés dans le tableau de la page suivante.

Les effets de 1'urée technique sont comparables à ceux obtenus avec l'urée pure.

L'administration d'urée pure, à la dose de $0, \mathrm{I} \mathrm{g} / \mathrm{kg}$ ne détermine aucune perturbation de la motricité gastrique. Pour les quantités supérieures, on observe une inhibition partielle ou totale de la motricité du rumen, après un temps de latence variable suivant la dose administrée et suivant 1'animal. L'inhibition est partielle pour les doses inférieures à $0,5 \mathrm{~g} / \mathrm{kg}$ et, en général, totale pour les doses supérieures. Pour cette quantité de $0,5 \mathrm{~g} / \mathrm{kg}$, on note sur 5 essais : 3 inhibitions partielles et 2 inhibitions totales.

L'inhibition porte à la fois sur la fréquence et l'amplitude des contractions, surtout pour les doses fortes, supérieures ou égales à $\mathrm{I} \mathrm{g} / \mathrm{kg}$.

I1 existe de nombreux facteurs de variation dont certains échappent à 1'analyse expérimentale. Dans les conditions où nous sommes placés, on peut éliminer le facteur alimentaire car l'alimentation et le mode d'administration de l'urée étaient comparables chez tous les animaux utilisés. De 


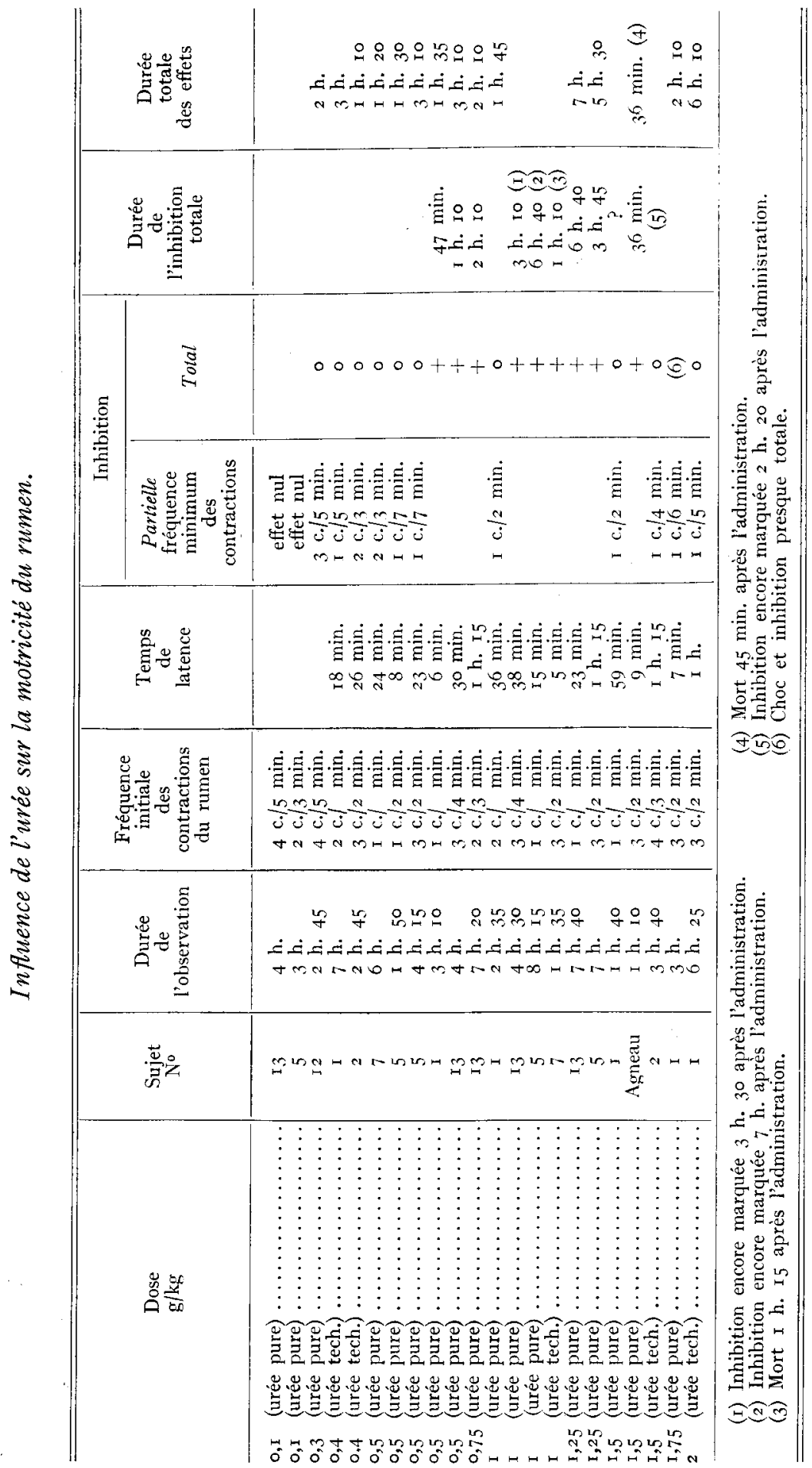


plus, la composition de la ration n'a pas varié pendant toute la durée de l'expérimentation et nous nous sommes efforcés d'effectuer les enregistrements de la motricité gastrique à la même heure de la journée.

2 essais consécutifs sur le même animal étaient séparés par un intervalle d'au moins 4 à 5 jours, ce qui élimine vraisemblablement l'intervention de la présence d'une quantité d'urée résiduelle dans le rumen.

Le cas du mouton $\mathrm{n}^{0} \mathrm{I}$ est particulièrement démonstratif et met en évidence la variabilité des résultats obtenus chez le même animal. Nous avons en effet observé :

\begin{tabular}{|c|c|}
\hline une inhibition totale à la & $0,5 \mathrm{~g} / \mathrm{kg}$, \\
\hline une inhibition partielle à la dose de. . . . . . . & $\mathrm{g} / \mathrm{kg}$ \\
\hline $\begin{array}{l}\text { une inhibition partielle à la dose de } \ldots \ldots \ldots \ldots \ldots \\
\text { une inhibition presque totale (une contraction toutes }\end{array}$ & $\mathrm{I}, 5 \mathrm{~g} / \mathrm{kg}$ \\
\hline
\end{tabular}

Le temps de latence observé entre le moment d'administration de l'urée et le début de l'inhibition des contractions est en général faible pour les doses élevées $(\mathrm{I}, 5 \mathrm{~g} / \mathrm{kg})$. Cependant, il ne dépend pas seulement de la quantité administrée : par exemple, dans le cas du mouton $n^{0} 5$, on note les résultats suivants :

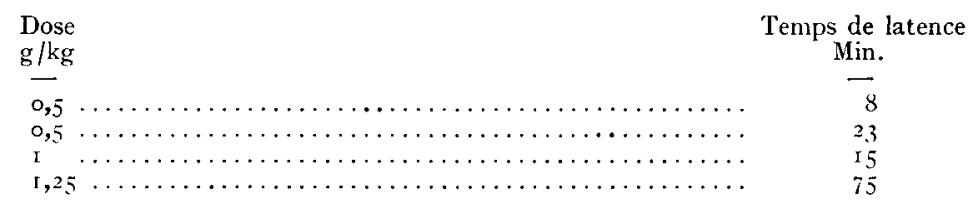

Il est vraisemblable que le degré de division de la masse alimentaire dans les réservoirs gastriques, conditionné par la mastication mérycique, intervient dans l'utilisation de l'urée par les microorganismes. Cette utilisation variable fait donc disparaittre plus ou moins rapidement une partie de l'urée ingérée, ce qui peut rendre compte des différences observées dans les résultats obtenus suivant les essais.

En conclusion, l'urée, administrée en solution aqueuse directement dans le rumen, détermine des perturbations de la motricité gastrique chez le mouton. Malgré de nombreuses variations dans les résultats obtenus, cette expérimentation permet de dégager les conclusions suivantes :

$I^{\circ} \mathrm{L}$ a dose de $\mathrm{O}, \mathrm{I} \mathrm{g} / \mathrm{kg}$ ne provoque aucune perturbation de la motricité gastrique.

$2^{0}$ La dose de $0,3 \mathrm{~g} / \mathrm{kg}$ entraîne une faible diminution de la fréquence des contractions.

$3^{\circ}$ L'inhibition de la motricité du rumen est partielle pour les doses comprises entre 0,3 et $0,5 \mathrm{~g} / \mathrm{kg}$.

$4^{\circ} \mathrm{A} 0,5 \mathrm{~g} / \mathrm{kg}$, suivant les animaux, l'inhibition est partielle ou totale.

$5^{\circ}$ Les doses supérieures à $0,5 \mathrm{~g} / \mathrm{kg}$ déterminent en général une inhibition totale de la motricité du rumen. I orsque le rumen n'est pas 
complètement paralysé, la fréquence de ses contractions est réduite d'au moins 4 fois et leur amplitude est diminuée.

$6^{\circ}$ La perturbation de la motricité gastrique s'observe après un temps de latence variant de 5 à 75 minutes, qui ne dépend pas obligatoirement de la quantité d'urée administrée.

$7^{\circ}$ Dans les conditions où nous nous sommes placés, nous n'avons pas noté de différence d'action entre l'urée pure et l'urée technique.

Du fait que l'administration d'urée est préconisée chez les ruminants pour augmenter l'efficacité protéique des rations, il était intéressant de compléter cette expérimentation par l'étude de l'influence de l'ingestion d'urée chez les animaux recevant une ration pauvre en protéines.

Pendant toute la durée des essais, les animaux ont reçu chaque jour la ration suivante:

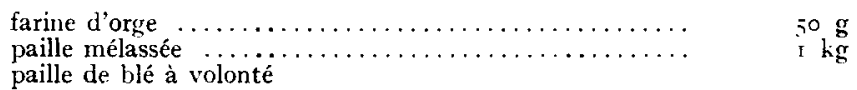

Cette ration n'apporte que 25 à $30 \mathrm{p}$. Ioo des protéines contenues dans le régime utilisé pour les premiers essais.

Le tableau suivant résume les résultats obtenus :

\begin{tabular}{|c|c|c|c|c|c|c|c|c|}
\hline \multirow[b]{2}{*}{$\begin{array}{l}\text { Dose } \\
\mathrm{g} / \mathrm{kg}\end{array}$} & \multirow[b]{2}{*}{$\underset{\text { Sujet }}{\text { Su }}$} & \multirow[b]{2}{*}{$\begin{array}{l}\text { Durée de } \\
\text { l'obser- } \\
\text { vation }\end{array}$} & \multirow[b]{2}{*}{$\begin{array}{l}\text { Fréquence } \\
\text { initiale } \\
\text { des } \\
\text { contractions } \\
\text { du rumen }\end{array}$} & \multirow[b]{2}{*}{$\begin{array}{l}\text { Temps } \\
\text { de } \\
\text { latence } \\
\text { min. }\end{array}$} & \multicolumn{2}{|c|}{ Inhibition } & \multirow[b]{2}{*}{$\begin{array}{l}\text { Durée } \\
\text { de } \\
\text { l'inhi- } \\
\text { bition } \\
\text { totale } \\
\text { min. }\end{array}$} & \multirow[b]{2}{*}{$\begin{array}{l}\text { Durée } \\
\text { totale } \\
\text { des } \\
\text { effets }\end{array}$} \\
\hline & & & & & $\begin{array}{l}\text { Partielle } \\
\text { fréquence } \\
\text { minimum } \\
\text { des con- } \\
\text { tractions }\end{array}$ & totale & & \\
\hline 0,10 & I & $3 \mathrm{~h}$ & $5 \mathrm{c.} / 4 \mathrm{~min}$. & & effet & & & \\
\hline 0,10 & ro & 3 h. Iо & 4 c. $/ 5 \mathrm{~min}$ & & effet & & & \\
\hline 0,25 & I & 5 h. Io & $3 \mathrm{c} / 2 \mathrm{~min}$. & & effet & & & \\
\hline 0,25 & Agneau & 2 h. +5 & 3 c. $/ 2 \mathrm{~min}$. & $i$ & & + & 28 & $2 \mathrm{~h}$. \\
\hline 0,25 & 10 & 3 h. 40 & 4 c. $/ 5 \mathrm{~min}$. & +6 & & + & 55 & 2 h. 20 \\
\hline 0,50 & I & 2 h. 20 & $3 \mathrm{c} . / 2 \mathrm{~min}$. & 7 & & + & 25 & I h. \\
\hline 0,50 & Agneau & 2 h. $4^{\circ}$ & c. $\min$. & I 2 & & + & 25 & 22.35 \\
\hline $0,5^{\circ}$ & 10 & 3 h. 10 & 3 c. 2 min. & 29 & & + & 80 & (I) \\
\hline
\end{tabular}

(I) La motricité est encore inhibée 2 h 40 après l'administration.

Les animaux recevant une ration pauvre en protéines sont donc plus sensibles à l'action de l'urée que les animaux recevant une alimentation normale. Dans les conditions oì nous nous sommes placés, nous observons toujours une inhibition totale de la motricité du rumen, même à faible dose : $0,25 \mathrm{~g} / \mathrm{kg}$ ( 2 fois sur 3 essais). Dans le cas des animaux recevant une ration normale, le même phénomène n'est observé qu'à la dose de $0,50 \mathrm{~g} / \mathrm{kg}$ ( 2 fois sur 5 essais).

Les recherches montrant1'efficacité de l'urée comme source de matières protéiques chez les ruminants mettent en évidence le fait que 1'utilisation de l'urée est augmentée si l'on remplace du mauvais fourrage par du 
fourrage de bonne qualité. Cette constatation indique donc la nécessité d'un certain apport de matières protéiques pour accroître la valeur alimentaire de l'urée. On peut donc penser que, dans nos conditions expérimentales, une partie de l'urée administrée ou une partie des sels ammoniacaux formés par uréolyse n'est pas utilisée par les microorganismes des réservoirs gastriques du fait de la carence protéique. Cette fraction inutilisée, plus importante que dans le cas des animaux soumis à une alimentation normale, serait responsable des perturbations accentuées de la motricité gastrique.

Pour vérifier cette hypothèse, nous avons injecté dans la circulation périphérique (veine jugulaire ou veine saphène externe) de moutons non anesthésiés porteurs d'une fistule permanente du rumen soit une solution d'urée soit une solution d'acétate d'ammonium. Ce dernier a été utilisé du fait de la production en grande quantité d'acide acétique au cours des fermentations microbiennes dans les réservoirs gastriques.

L'enregistrement de la motricité du rumen a été réalisé selon la technique déjà décrite (5). Nous avons obtenu les résultats suivants.

\section{Action de l'injection intra-veineuse d'urée.}

L'injection rapide de solution d'urée, en 20 à 60 secondes, à des doses variant de $0, \mathrm{I}$ à $\mathrm{ro} \mathrm{cg} / \mathrm{kg}$ ne détermine aucune perturbation de la motricité gasrique. Par contre des doses plus fortes : 15 à $40 \mathrm{cg} / \mathrm{kg}$ injectées lentement en goutte à goutte à des vitesses variant de $I_{5}$ à $40 \mathrm{cg} /$ minute, déterminent une hypermotricité caractérisée dans tous les cas par une augmentation de l'amplitude des contractions et fréquemment par un accroissement de leur rythme de l'orde de 30 à $40 \mathrm{p}$. Ioo.

\section{Action de l'injection intra-veineuse d'acétate d'ammonium.}

Comme dans le cas de l'urée, nous avons recherchél'influence d'une injection rapide et d'une injection lente.

Lorsque l'injection est rapide, on observe une inhibition fugace à la dose de $0, \mathrm{I} \mathrm{cg} / \mathrm{kg}$, totale pendant 5 à 6 minutes à la dose de $5 \mathrm{cg} / \mathrm{kg}$.

En injection lente, en goutte à goutte, à des vitesses variant de 5 à $20 \mathrm{cg} /$ minute, on note un ralentissement marqué de la fréquence des contractions aux doses de 5 à $\mathrm{Io} \mathrm{cg} / \mathrm{kg}$ et une paralysie totale du rumen à la dose de $20 \mathrm{cg} / \mathrm{kg}$. La motricité reprend peu à peu ses caractères initiaux à la fin de l'injection; la durée totale des effets inhibiteurs varie suivant les animaux de I heure à $2 \mathrm{~h}$. 40. On observe parfois une hypermotricité secondaire caractérisée par une augmentation de l'amplitude des contractions.

Ces observations conduisent à penser que l'inhibition de la motricité du rumen, observée chez le mouton à la suite de l'ingestion d'urée, n'est 
pas due à l'action propre de cette substance, mais résulte du passage dans le sang des produits de l'uréolyse microbienne et plus particulièrement des sels ammoniacaux.

L'urée qui est également résorbée par la muqueuse du rumen ne peut être tenue pour responsable de l'arrêt de la motricité. En effet son administration lente par voie veineuse détermine au contraire une hypermotricité. Cette constatation est à rapprocher du fait que l'urée est transformée en matières protéiques dans les réservoirs gastriques et non pas éliminée en grande partie soit sous la forme d'urée soit sous la forme de sels ammoniacaux comme les possibilités d'absorption du rumen le laisseraient à priori supposer.

Ces observations nous ont conduit à émettre l'hypothèse selon laquelle l'urée et les sels ammoniacaux du sang doivent pouvoir être excrétés dans les réservoirs gastriques, ceux-ci se comportant comme un véritable émonctoire.

Pour vérifier cette hypothèse, nous avons utilisé deux méthodes : I $^{\mathrm{o}}$ Etude du passage dans le rumen d'urée administrée lentement en goutte-à-goutte intra-veineux.

$2^{0}$ Perfusion du rumen en place par du sang enrichi en urée.

\section{Administration d'urée par voie veineuse en goutte à goutte.}

La technique expérimentale est la suivante: sur un mouton anesthésié au chloralose, le rumen-réseau et la caillette sont vidés de leur contenu, lavés et séparés du reste du tube digestif par des ligatures. On introduit ensuite 2 litres de liquide de Tyrode à $3^{8^{\circ} \mathrm{C}}$ non glucosé dans le rumen-réseau et $250 \mathrm{~cm}^{2}$ dans la caillette. Un premier prélèvement (I) de sang, de salive mixte et de contenu du rumen-réseau et de la caillette est effectué une demi-heure plus tard. Puis l'urée, en solution à $0,200 \mathrm{~g} / \mathrm{cm}^{3}$ et à la dose de $0,200 \mathrm{~g} / \mathrm{kg}$ est administrée par voie veineuse en goutte-à-goutte. Un deuxième prélèvement (2) est réalisé à la fin de l'injection et un troisième (3) une heure plus tard.

Les dosages d'urée (méthode de Conway) dans les prélèvements ont donné les résultats suivants :

Mouton no 1,5.5 kg. Durée de l'injection : 55 min.

\begin{tabular}{|c|c|c|c|}
\hline & & rée e & \\
\hline & I & 2 & 3 \\
\hline & - & - & - \\
\hline Sang artériel ......... & 245 & $34 \mathrm{I}$ & 453 \\
\hline Salive mixte $\ldots \ldots \ldots \ldots$ & 210 & 26 I & 376 \\
\hline Rumen-réseau ......... & 20 & 29 & 22 \\
\hline Caillette $\ldots \ldots \ldots \ldots \ldots$ & 36 & 46 & 65 \\
\hline
\end{tabular}


Mouton $n^{0} 2,33 \mathrm{~kg}$. Durée de l'injection : $25 \mathrm{~min}$.

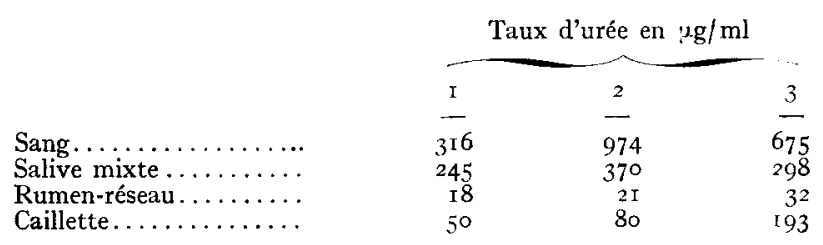

Mouton $n^{\circ} 3,40 \mathrm{~kg}$. Durée de l'injection : $42 \mathrm{~min}$.

\begin{tabular}{|c|c|c|c|}
\hline & & arée e & \\
\hline & I & 2 & 3 \\
\hline & - & - & - \\
\hline Sang......... & 344 & 652 & 442 \\
\hline Salive mixte $\ldots \ldots \ldots \ldots$ & $2 \mathrm{II}$ & $35^{8}$ & 442 \\
\hline Rumen-réseau . . . . . . . . & 33 & 50 & 78 \\
\hline Caillette $\ldots \ldots \ldots \ldots \ldots$ & 43 & 92 & 148 \\
\hline
\end{tabular}

Ces constatations montrent donc que l'urée du sang peut revenir dans les réservoirs gastriques par deux voies : la salive et le passage à travers la muqueuse gastrique. L'urée qui est excrétée par la caillette peut être tenue comme étant perdue par l'animal.

\section{2. - Periusion du rumen.}

Sur un mouton anesthésié au chloralose, le rumen-réseau est vidé et le cardia et l'orifice réseau-feuillet sont ligaturés. On introduit dans la cavité 2 litres de liquide de Tyrode à $3^{8^{\circ} \mathrm{C}}$ non glucosé. Des ligatures vasculaires permettent d'exclure totalement du point de vue circulatoire le rumen-réseau du reste de 1'organisme. Ia perfusion est réalisée au moyen d'une pompe dont le débit est réglé de façon à obtenir une pression artérielle de ro à $\mathrm{I} 2 \mathrm{~cm}$ de mercure. Le sang veineux est oxygéné dans l'appareil de BoulANGER et GrifFie (6). Ce dispositif permet d'effectuer la perfusion avec $400 \mathrm{~cm}^{3}$ de sang hépariné enrichi en urée $(\mathrm{I} \mathrm{g} / \mathrm{l})$.

Les dosages d'urée dans le sang et dans le contenu du rumen-réseau ont donné les résultats suivants :

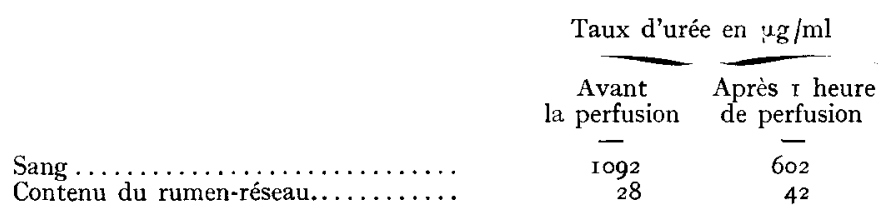

Nous avons vérifié que le taux d'urée d'un prélèvement de sang, maintenu dans les mêmes conditions de température et d'oxygénation mais ne circulant pas dans le rumen, n'était pas modifié.

On observe donc une disparition de l'urée sanguine et un enrichisse- 
ment du contenu du rumen-réseau. Ce fait ne peut s'expliquer que par 1'excrétion d'urée à travers la paroi du rumen.

Ces constatations mettent donc en évidence le cycle de l'urée, administrée par voie buccale chez les ruminants. L'urée résorbée au niveau du rumen, peut revenir dans ce réservoir par deux voies : la sécrétion salivaire et l'excrétion à travers la muqueuse gastrique. Ces constatations apportent une contribution à la connaissance des modalités de l'utilisation de l'urée dans l'alimentation des ruminants et mettent en évidence l'existence d'un mécanisme de "récupération " qui permet à l'animal d'utiliser 1'urée déjà résorbée au niveau des réservoirs gastriques.

Une expérimentation actuellement en cours, laisse supposer queles sels ammoniacaux subissent la même destinée.

\section{Reçu pour publication le I9 septembre I958.}

\section{RÉFÉRENCES BIBLIOGRAPHIQUES}

(I) Dinning (J. S.), Briggs (H. M.), Gailup (W. A.), On (H. W.) etButler (R.). - Eiffects of orally administred urea on the ammonia and urea concentration in the blood of Cattle and Sheeps with observation in blood ammonia levels associated with symptoms of alkalosis. Am.J. Physiol., 153, $4 \mathrm{I}-46$, I 948 ,

(2) Ciark (R.), OyaerT (W.) et Quin (J. I.). - Studies on the alimentary tract of the Merino Sheep in South Africa. XXI. - The toxicity of urea to Sheep under different conditions. Onderstepoort J. Veter. Res., 25, 73-78, r951.

(3) Fujimoto (Y.) et Tajima (M.). - Pathological studies on urea poisoning. Jap. J. Veter. Sci., 15, I25-I32, I953.

(4) Thomas (H.), Bullington (B. S.), Byrd (C. E.) et Harris (T. W.). Urea poisoning in the Bovine. North. Am. Vete., 36, I07-I09, I955.

(5) Le BARS (H.), Nitescu (R.) et Simonnet (H.). - Recherches sur la motricité du rumen chez les petits Ruminants. I. - Motricité normale. Bull. Acad. Vét., 26, 287-300, I953.

(6) Boulanger (P.) et Griffie (R. A.). - Appareil à perfusion fonctionant avec un volume de liquide restreint. $J$. Physiol., 40, 127-x28 A, x948.

Une subvention accordée par O.N.I.A. (Toulouse) a facilité la réalisation de ce travail.

Le Directeur-Gérant : B. LACLAVIÈRE.

I.N.R.A

BIBLIOTHEQUE UD 399g0 gie Bussière à Saint-Amand (Cher), France. - 6-2-1959)

DOMAINE DE CROUELépót légal: 1 1 trimestre $1959 \quad N^{\text {to }}$ d'impression: 269

63039

CLERMQNT-FD CEDEX? 